\title{
THE NONAGENARIAN NEUTRINO
}

\author{
C Sivaram $^{1}$, Arun Kenath ${ }^{* 2,3}$, Kiren $\mathrm{OV}^{2}$
}

\begin{abstract}
December 4, 2020 marks 90 years since the concept of neutrinos was proposed as a consequence of the observed discrepancies in several experiments on radioactive decays of various isotopes. There have been great many developments in our understanding of this elusive particle over the past nine decades, also leading to several Nobel Prizes awarded to work on neutrino physics. But there are many aspects of the neutrinos that are still not completely understood, including even its actual rest mass. The neutrino still remains an enigma and we have yet to learn a lot about its different properties. This article summarises the overall picture of the current understanding of the neutrino, right from its inception.
\end{abstract}

Keywords: Neutrino; neutrino detection; beta decay

\footnotetext{
${ }^{1}$ Indian Institute of Astrophysics, Bengaluru, 560034, Karnataka, India

${ }^{2}$ Department of Physics and Electronics, CHRIST (Deemed to be University), Bengaluru, 560029, Karnataka, India

${ }^{3}$ Department of Physics, Christ Junior College, Bengaluru, 560029, Karnataka, India

*Corresponding author: kenath.arun@cjc.christcollege.edu (Arun Kenath)

sivaram@iiap.res.in (C Sivaram); kiren.ov@res.christuniversity.in (Kiren OV)
} 


\section{Introduction}

The concept of the neutrino was envisaged just ninety years ago, when Wolfgang Pauli wrote his famous letter, addressed to 'Dear Radioactive Ladies and Gentlemen,' on December 4, 1930 [1]. It was addressed to the participants of a conference convened to discuss a crisis situation evoked by several experiments on radioactive decays of various isotopes. It was found that the electrons emerging from the beta decay had a range of energies, which led to contradictions with the very fundamental physical laws of conservation of energy and momentum. The energy associated with the decaying particle did not match the energies of the product particles, implying that some energy (momentum) was missing. The crisis was serious enough for even stalwarts like Niels Bohr to question whether energy conservation is violated in beta decay. Pauli felt that such an extreme step to resolve the issue was absurd and instead postulated the existence of a new neutral particle (presumably having no rest mass or electric charge) carrying away this missing energy. It was this bold proposal which was contained in Pauli's letter to the conference participants, which he himself was unable to attend.

Pauli himself did not name the particle as neutrino. The neutron itself was yet to be experimentally discovered and this was done by Chadwick in 1932. Soon Enrico Fermi gave a detailed theory for the beta decay process (for e.g. the decay of a neutron to a proton and electron) which involved interaction of two currents, later known as a four-fermion interaction [2]. The fourth fermion here was yet to be discovered and Fermi dubbed it the neutrino (or small neutron) as it was also expected to be a neutral particle with near zero rest mass. So Fermi's universal theory of beta decay naturally accommodated Pauli's postulated particle which was now dubbed the neutrino, yet to be discovered, but carrying away the missing energy in beta decay.

It became clear that it would be very difficult to detect such a light neutral particle. The cross section for its interaction with other particles (say nuclei) was estimated to be a miniscule $10^{-48} \mathrm{~m}^{2}$ (from Fermi's universal weak interaction constant, $G_{F}$ ) [3]. This implied that neutrinos could traverse even light-years of lead without interaction. It is not a surprise that the idea of such a ghostly particle took some time for physicists to readily accept and discuss the implications. In this context, it is interesting that Hans Bethe when he proposed the famous CNO cycle [4] as the source for thermonuclear reactions powering the Sun and other stars, did not mention the neutrino although the set of reactions involves two beta decays of the nuclei $N_{13}$ and $O_{15}$. So also the proposed pp cycle (proton-proton fusion) initially ignored the neutrinos. However, it was soon realised that these set of thermonuclear reactions taking place 
in the core of the Sun, should lead to the emission of $2 \times 10^{38}$ neutrinos per second. This would imply a flux on Earth of about $7 \times 10^{10} \mathrm{~cm}^{-2} \mathrm{~s}^{-1}$. In other words one quadrillion solar neutrinos pass through each of us every second [5].

\section{Detection of neutrinos}

How could we detect such weakly interacting particles, most of which would go right through the earth? Gamow in particular realised that evolved stages of a star when it starts fusing nuclei of heavier elements, should lead to catastrophic prodigious neutrino production, which would rapidly drain away energy from the stellar interior. As he was in Brazil at that time, he named it the Urca process after a gambling den where money was being rapidly drained away [6]. It was realised later that several other neutrino producing processes could be involved at such high temperatures (of a few billion degrees) when heavier nuclei were synthesised such as the photoneutrino and neutrino pair production processes. These rapid energy draining mechanisms (as neutrinos come out right through the star) reduce the duration of the silicon burning reactions producing iron in the stellar core to a few days. Finally, when a massive star produces iron in its core, the thermonuclear reactions halt, as iron has the maximum nuclear binding energy. The massive stellar core collapses to form a neutron star. The conversion of nuclei to neutrons, dubbed inverse beta decay, releases in a few seconds, something like $10^{58}$ neutrinos. The forming neutron star is heated up to a trillion degrees or so, and most of the energy is radiated away as neutrinos [7].

That all this is not just an imaginative sophisticated science story, but actually occurs in nature, was spectacularly demonstrated in February 1987, when the supernova SN 1987A, caused by the collapse of a massive star (at least 10 solar mass) occurred in the LMC galaxy (neighbouring the Milky Way), about 50 kiloparsec away. The Kamiokande water detector in Japan and the IMB detector in the US, registered about a dozen events in a few seconds, which is the neutrino flux expected at the Earth when such a massive star collapses to form a neutron star (in the LMC) [8].

The number of neutrino events is estimated by the product of the flux, the neutrino interaction cross section, and the number of atoms in the detector. As the neutrinos interact very weakly, very large detectors, in this case, having several kilotons of water are required to detect a few events. It was gratifying that the total estimated energy lost by the collapse in the form of neutrinos (as implied by the flux detected on Earth) matches the gravitational binding 
energy of $3 \times 10^{53} \mathrm{ergs}$ of a corresponding $1.4 M_{\odot}$ neutron star. In fact such a remnant NS was recently detected in the SN 1987A debris [9].

The Kamiokande detector [10] was pioneered by Masatoshi Koshiba (shared half the Nobel Prize in physics with Raymond Davis Jr. in 2002), who passed away recently on November 12, 2020, at the age of 94 . The Kamiokande detector also confirmed the solar neutrino deficit first observed by Davis in his Homestake detectors [11]. Davis realised early, sixty years ago, that very large detectors are needed to capture a significant number of solar neutrinos and used a million gallons of tetrachloroethylene (a dry cleaning fluid) in the deep underground mine at Homestake. He was surprised that the number of neutrinos detected were only about a third of that expected from the solar flux. This was confirmed in the 1980s, by the Kamiokande detector which was sensitive to the higher energy neutrinos coming from the $B_{8}$ reaction in the Sun [12].

The very first detection of neutrinos was from nuclear fission power reactors. However, unlike the fusion reactions in the Sun's core, the nuclear fission reactions produce antineutrinos. A gigawatt reactor produces a flux of several quadrillion antineutrinos. These were detected in the Savannah reactor in 1956, by Reines and Cowan [13]. Davis realised that solar neutrino detection is different than the reactor antineutrino detection. Meanwhile it was discovered that there is another neutrino associated with the muon, the heavier cousin of the electron. The muon neutrino was detected in 1962.

Later the tau lepton, a still heavier cousin, 17 times the muon mass was discovered. So there is also a tau neutrino associated with this heavier lepton. The threshold energy for the pp neutrinos (produced in the pp fusion reactions) are too small to be detected in either the Davis or Kamiokande experiment as their energy is only $0.4 \mathrm{MeV}$. So the gallium experiments, GALLEX and SAGE were set up, using gallium as the detector material. These actually detected the much higher flux from the low energy pp neutrinos [14].

\section{Solar neutrino problem}

But still the puzzle of the deficit of solar neutrinos remained. As there were other neutrino flavours, it was proposed by Pontecorvo, Maki, Nakagawa, Sakata and others that neutrino oscillations could occur, wherein say a beam of pure electron neutrinos after traversing some distance could become a mixture involving other flavours (the flavour state being a combination of mass Eigen states, a typical quantum phenomenon) $[15,16]$. The solar neutrino experiments like those of Davis or Kamiokande could detect only the electron neutrinos. 
However, McDonald and Kajita, (student of Koshiba) proposed that heavy water if used as a detector could also detect the other flavour neutrinos, through what are known as neutral current interactions. So the Sudbury Neutrino Observatory, using kiloton of heavy water was designed in an underground mine in Canada. Indeed the other neutrino flavours produced by the oscillations were detected (by the neutral current interactions) [17]. When the fluxes of these detected additional flavours were added up, the expected solar neutrino flux was obtained. This remarkable result earned a physics Nobel Prize in 2015.

Again neutrino oscillations involving atmospheric neutrinos were detected by upgraded Super-Kamiokande. This explained why atmospheric muon neutrinos (from decay of pions) are seen in fewer numbers than expected [18]. Tellurian neutrinos and antineutrinos from decay of radioactive isotopes in the Earth's interior have also been detected by KamLAND [19]. It is also still not established whether neutrinos could be Majorana particles.

\section{Astrobiological implications of neutrinos}

Neutrinos of all flavours are expected to be produced copiously in the big bang in the first one second. They should now form a background - like the microwave background which is the fossil remnant of the hot radiation (high energy radiation) which characterised the hot dense phase of the earlier epoch (cooling with expansion) - but with a temperature of around $2^{0} \mathrm{~K}$. Despite several suggestions and proposed experiments, this cosmic neutrino background has not yet been detected. The fossil remnant of neutrinos will also form a background with an estimated density of about 150 per cubic centimetre, per species. So summed over all the six species we expect a fossil neutrino background with a number density of one thousand per cubic centimetre.

So if each neutrino had a mass of about even twenty electron volts, this would imply that the Universe would have a density much greater than the closure density and would have collapsed several billion years ago. Most definitely a Universe where a neutrino had a fifty electron volt rest mass (still ten thousand times lower than the electron mass which is hitherto the lightest known elementary particle) would not have had much chance to develop biological life, let alone have advanced forms of evolved life. Since at present we do not have a definitive understanding of neutrino masses (the standard model says it should be zero) one wonders whether there can be some anthropic requirement for the low masses.

Neutrinos are known to be produced prodigiously in a Type-2 supernova, where a massive star collapses after its iron core can no longer continue to produce thermonuclear energy. The core evolves to form a superhot neutron star. The neutrino flux on earth from 
SN1987A, caused by a massive star collapse in the LMC, sixty kilo-parsecs away, was something like hundred trillion per square metre. Ten events were recorded in the Kamiokande and IMB detectors, which is what was expected for a one kiloton water detector. These neutrinos are of several mega electron volt energy. Betelgeuse, 400 light years away is a candidate for a Type- 2 supernova. It may explode in a few thousand years. In such a case, a kiloton detector would experience millions of such neutrino capture events. Typically each one of us would capture a hundred high energy neutrinos.

A supernova occurring ten light years away would produce ten billion neutrino captures in a kiloton detector and each of us would capture one lakh high energy neutrinos which could potentially cause DNA cell damage. The captured neutrinos would produce damaging gamma rays, high energy neutrons, among other things. So life on potential planets neighbouring massive star associations (or stellar systems) like OB associations could be severely constrained.

\section{Conclusion}

Although Pauli as the initial proposer, missed a Nobel (he got it in 1945 for explaining atomic stability with his exclusion principle), several Nobel Prizes have been awarded for discoveries involving neutrinos. Despite more than 60 years after its actual discovery in 1956, many properties of the neutrinos are still unknown. Confirmation of neutrino oscillations gives only the difference in mass squared. The actual masses of the different neutrino types is still unknown. There are limits from cosmology (on the sum of the masses) and from neutrinoless double $\beta$ decay experiments. These along with the KATRIN, Tritium decay experiment, give masses of around an $\mathrm{eV}$ or less [20].

The neutrino is still an enigma, and so many experiments worldwide both on going and proposed are underway to unravel its mysteries, from DUNE to Daya Bay Reactor Neutrino Experiment, not to forget ICECUBE, in the Antarctic which in recent years detected a few ultrahigh energy neutrino. 90 years after it was postulated it remains an enigma and we have yet to learn a lot about its different properties and manifestations.

\section{References:}

1. Brown, L. M., The idea of the neutrino. Physics Today, 1978, 31, 23-28.

2. Wilson, F. L., Fermi's Theory of Beta Decay. American Journal of Physics, 1968, 36, $1150-1160$.

3. Bahcall, J. N., Neutrino Astrophysics, Cambridge University Press, Cambridge, 1989. 
4. Bethe, H. A., Energy Production in Stars, Physical Review, 1939, 55, 541-547.

5. Bellerive, A., Review of solar neutrino experiments, International Journal of Modern Physics A, 2004, 19, 1167-1179.

6. Gamow, G., My World Line: An Informal Autobiography, Viking Press, New York, 1970, p. 137.

7. Janka, H-Th., Neutrino Emission from Supernovae In Handbook of Supernovae (eds. Alsabti A. and Murdin P.), Springer, Cham., 2017, pp. 1575-1604.

8. Arnett, W. D. et al., Supernova 1987A, Annual Review of Astronomy and Astrophysics, 1989, 27, 629-700.

9. Cigan, P. et al., High Angular Resolution ALMA Images of Dust and Molecules in the SN 1987A Ejecta, Astrophysical Journal, 2019, 886, 51.

10. Fukuda, S. et al., The Super-Kamiokande detector, Nuclear Instruments and Methods in Physics Research A, 2003, 501, 418-462.

11. Cleveland B. T. et al., Measurement of the Solar Electron Neutrino Flux with the Homestake Chlorine Detector, Astrophysical Journal, 1998, 496, 505-526.

12. Bahcall, J. N. et al., How uncertain are solar neutrino predictions?, Physics Letters $B$, 1998, 433, 1-8.

13. Reines, F. and Cowan Jr., C. L., The Neutrino, Nature, 1956, 178, 446-449.

14. Abdurashitov, J. N. et al., Measurement of the solar neutrino capture rate with gallium metal. III. Results for the 2002-2007 data-taking period, Physical Review C, 2009, 80, 015807

15. Pontecorvo, B., Mesonium and anti-mesonium. Sov. Phys. JETP, 1957, 6, 429-431.

16. Maki, Z et al., Remarks on the Unified Model of Elementary Particles, Progress of Theoretical Physics, 1962, 28, 870.

17. Ahmad, Q. R. et al., Measurement of the rate of $v_{\mathrm{e}}+\mathrm{d} \rightarrow \mathrm{p}+\mathrm{p}+\mathrm{e}^{-}$interactions produced by ${ }_{8} \mathrm{~B}$ Solar neutrinos at the Sudbury Neutrino Observatory, Physical Review Letters, 2001, 87, 071301.

18. Fukuda, Y. et al., Evidence for Oscillation of Atmospheric Neutrinos, Physical Review Letters, 1998, 81, 1562-1567.

19. Gando, A. et al., Partial radiogenic heat model for Earth revealed by geoneutrino measurements, Nature Geoscience, 2011, 4, 647-651.

20. Angus, G. W. et al., On the Proof of Dark Matter, the Law of Gravity, and the Mass of Neutrinos, Astrophysical Journal Letters, 2007, 654, L13-L16. 